\title{
Fabrication and Calibration of Directionally-Resolved Micropillars Using Off-the-Shelf Piezoresistive Dies
}

\author{
Xiaozhi Kong, Arthur Poulain, Paul Nathan, and David M. Birch
}

\begin{abstract}
A two-component micropillar system has been developed for use in flow characterization and control applications. It is demonstrated that the piezoresistive elements in a conventional pressure sensing die can be used to measure moments directly applied to the die with reasonable sensitivity. The concept is then demonstrated by bonding a small, rigid pillar to the centre of a commercially-available, off-the-shelf doped silicon pressure sensing membrane with integrated piezoresistive bridge elements. The signal response of the system is then calibrated against aerodynamic loading, and a typical sensitivity of $70 \mathrm{mV} / \mu \mathrm{Nm}$ is demonstrated. The functionality of the micropillars in flow control applications is verified by recessing the pillar below a flat surface on which a model boundary layer is developed. By processing the signals through the membrane bridge-arms independently, directionally-resolved forces may also be obtained.
\end{abstract}

\section{Introduction}

$\mathrm{T}$ HE accurate measurement of aerodynamic wall friction is critically important in the experimental characterization of wall-bounded turbulence, as it is one of the fundamental scaling parameters. It is also, possibly, one of the most difficult quantities to measure in these flows: the values are small, time-dependent, two-dimensional and spatially-varying $(1 ; 2)$, red and the ability to resolve shear fields in both space and time is becoming increasingly important (3). Wall friction is typically expressed in the form of a wall shear velocity $u_{\tau}=(\tau / \rho)^{1 / 2}$ (where $\tau$ is the dimensional wall shear stress and $\rho$ is the fluid density). Commonly, time-averaged values of $u_{\tau}$ are inferred from the mean velocity profiles near the wall, using (for example) Preston tubes (4) or pulsed-wires (5)). The velocity profile is then used to infer wall shear by employing well-accepted empirical look-up tables $(6 ; 7)$. For the particular case of turbulent boundary layers, this technique requires certain assumptions about the scaling of these flows. Because these assumptions are now under investigation and are being questioned $(8 ; 9)$, alternative, direct techniques for measuring wall shear are needed.

X. Kong is with the School of Naval Architecture \& Ocean Engineering, Dalian Maritime University, Dalian, Liaoning, China.

A. Poulain is with ENSTA ParisTech, 828, boulevard des Maréchaux, 91120 Palaiseau, France.

P. Nathan is with Surrey Sensors Ltd., Unit 13 Parklands, Railton Road, Guildford, Surrey, GU2 9JX, UK.

D. M. Birch is with the University of Surrey, Department of Mechanical Engineering Sciences, Guildford, Surrey, GU2 7XH, UK; e-mail:d.birch@surrey.ac.uk.
Micropillars are an attractive technology for the measurement of wall shear. These devices consist of small, high aspect ratio columns which protrude into the flow, remaining within universal logarithmic region or even the viscous sublayer of a turbulent boundary layer (which will typically be less than $100 \mu \mathrm{m}$ thick in typical laboratory applications). The deflection of the micropillar is then a unique function of the near-wall fluid velocity profile and the mechanical properties of the pillar itself. Because the relationship between the velocity profile and wall shear is known, the wall shear can be obtained from the pillar deflection by empirical calibration, or even derived analytically if the velocity profile is assumed to be universal. The pillar deflections can be measured either optically $(10 ; 11 ; 12)$, requiring high-speed cameras, light sources and additional instrumentation mounted outside of the flow facility, or by using strain sensors integrated into the pillars themselves $(13 ; 14)$. For flow control purposes, the latter remains preferable.

There have been significant recent advancements in the repeatable fabrication of these micropillar structures from soft materials $(15 ; 16)$, which offer improved sensitivity and robustness compared to MEMS devices (17). Nevertheless, the fabrication of these micropillars remains resource-intensive, and the cost of production precludes the fabrication of very-large, performance-consistent arrays which are needed for spatial characterizations and the mapping of large, near-wall turbulence structures known to be strongly associated with friction drag (see (18)). Similarly, any practical boundary layer control efforts would require arrays of sensors with spacing sufficiently small to resolve the near-wall structures (typically 100 wall units) and an area coverage of the same order as the integral length scale.

To address this problem, a micropillar system has been developed using a re-purposed, commercially-available miniature doped silicon pressure sensing die with embedded piezoresistive strain sensors. Off-the-shelf, massmarket sensors are attractive for this application as they are inexpensive, mature technologies.

II. Modeling the effect of a micropillar on a conventional piezoresistive bridge membrane

Using a piezoresistive pressure die as a micropillar sensor will result in off-design loads and strains. The response of a typical die (and its sensing elements) to 
this loading condition must therefore first be assessed by comparing the magnitude and sense of the resultant surface strains to those encountered in on-design loading conditions.

\section{A. Response of a die diaphragm to an applied pressure}

Consider a typical square pressure sensing die. A nominally square area in the centre of the die (having edge length $a$ ) is typically etched away from one face to leave a thin membrane of silicon which will deform measurably under the application of pressure. If it is assumed that the membrane thickness $h \ll a$, the applied pressure $P$ is uniform and constant, the material is homogeneous and isotropic and the deformations are small relative to $a$, then the out-of-plane displacement profile $w(x, y)$ of the membrane is given by the thin-plate equation,

$$
\frac{\partial^{4} w}{\partial x^{4}}+2 \frac{\partial^{4} w}{\partial x^{2} \partial y^{2}}+\frac{\partial^{4} w}{\partial y^{4}}=\frac{P}{D}
$$

where $x$ and $y$ are the Cartesian coordinates in the plane of the membrane, and $D$ is the bending stiffness of the membrane, such that

$$
D=\frac{h^{3} E}{12\left(1-v^{2}\right)}
$$

where $E$ is the elastic modulus of the material and $v$ is the Poisson ratio. For a square diaphragm subject to Cauchy boundary conditions, the solution to eq. (1) is known to be

$$
\begin{aligned}
w(x, y) & =\frac{2 P a^{4}}{\pi^{5} D} \sum_{j} \frac{(-1)^{\frac{j-1}{2}} \cos \left(\alpha_{x}\right)}{j^{5} \cosh \left(\alpha_{j}\right)}\left[2 \cosh \left(\alpha_{j}\right)-\left(2+\alpha_{j} \tanh \left(\alpha_{j}\right)\right) \cosh \left(\alpha_{y}\right)+\alpha_{y} \sinh \left(\alpha_{y}\right)\right] \\
& +\frac{a^{2}}{2 \pi^{2} D} \sum_{j} \frac{(-1)^{\frac{j-1}{2}} \cos \left(\alpha_{x}\right)}{j^{2} \cosh \left(\alpha_{j}\right)} A_{j}\left[\alpha_{j} \tanh \left(\alpha_{j}\right) \cosh \left(\alpha_{y}\right)-\alpha_{y} \sinh \left(\alpha_{y}\right)\right] \\
& +\frac{a^{2}}{2 \pi^{2} D} \sum_{j} \frac{(-1)^{\frac{j-1}{2}} \cos \left(\alpha_{y}\right)}{j^{2} \cosh \left(\alpha_{j}\right)} A_{j}\left[\alpha_{j} \tanh \left(\alpha_{j}\right) \cosh \left(\alpha_{x}\right)-\alpha_{x} \sinh \left(\alpha_{x}\right)\right]
\end{aligned}
$$

where $j=1,3,5, \ldots$ and the substitutions $\alpha_{j}=j \pi / 2$, $\alpha_{x}=j \pi x / a$ and $\alpha_{y}=j \pi y / a$ have been made for convenience. The constants $A_{j}$ are defined by the solution to the system

$$
\begin{aligned}
& \frac{\pi A_{j}}{j}\left(\frac{\alpha_{j}}{\cosh ^{2}\left(\alpha_{j}\right)}+\tanh \left(\alpha_{j}\right)\right)+8 j \sum_{i} \frac{A_{i}}{i^{3}}\left(1+\frac{j^{2}}{i^{2}}\right)^{-2} \\
= & \frac{4 P a^{2}}{\pi^{2} j^{4}}\left(\frac{\alpha_{j}}{\cosh ^{2}\left(\alpha_{j}\right)}-\tanh \left(\alpha_{j}\right)\right)
\end{aligned}
$$

where the index $i=1,3,5, \ldots$; the plane stresses $\sigma_{x}$ and $\sigma_{y}$ on the surface of the membrane may then be obtained from $w(x, y)$ as

$$
\begin{aligned}
& \sigma_{x}(x, y)=-\frac{6 D}{h^{2}}\left(\frac{\partial^{2} w}{\partial x^{2}}+v \frac{\partial^{2} w}{\partial y^{2}}\right) \\
& \sigma_{y}(x, y)=-\frac{6 D}{h^{2}}\left(\frac{\partial^{2} w}{\partial y^{2}}+v \frac{\partial^{2} w}{\partial x^{2}}\right)
\end{aligned}
$$

with the maxima occurring near the boundaries.

Pressure sensing dies are typically designed to respond to the strain (or, equivalently, $\sigma_{x}$ or $\sigma_{y}$ ) around the edges of the membrane, where the maximum stress is expected to be encountered. This is usually done through the use of a Wheatstone bridge of strain-sensitive resistances (see
Figure 1). Knowing the full-scale range of the sensing die, then, eqs. (3) and (5) can be used to approximate the dimensional range of sensitivity of the resistances, noting that both $\sigma_{x}$ and $\sigma_{y}$ are independent of $D$, while $a$ (and, to a lesser extent, $h$ ), can be readily measured. Conveniently, Timoshenko (19) showed that the maxima of the solution to eqs. (3) and (5) can be reasonably-well approximated by

$$
\max \left(\sigma_{x}\right)=\max \left(\sigma_{y}\right) \approx 0.3102\left(\frac{a}{h}\right)^{2} P
$$

B. Response of a die diaphragm to an applied moment

Next, consider the case of a pressure sensing die with a square cylinder of side length $d$ and height $l$ attached to its surface at the centre, as illustrated in figure 2 (note that a square cylinder is being considered simply for the purposes of mathematical convenience.) If a force is applied to the cylinder normal to its axis, the die will be subjected to an applied shear force and bending moment. If the effects of the shear are assumed to be negligible (see (19)), the moment caused by the pillar can be approximated by an equivalent distributed, zero-mean linear load over the area $-d / 2 \leq x \leq d / 2,-d / 2 \leq y \leq d / 2$. For simplicity, if it is assumed that a total moment $M$ about the $y$-axis is distributed uniformly within $-d / 2 \leq x \leq d / 2$ (equivalent to the assumption that $d \sim a$ along $\bar{y})$, then an analytical solution for $w(x, y)$ exists as

$$
\begin{aligned}
w(x, y) & =\frac{6 M a^{3}}{d^{2} D} \sum_{k} \frac{\beta_{k}(-1)^{\frac{k}{2}}}{\cosh \left(\alpha_{k}\right)}\left[2 \cosh \left(\alpha_{k}\right)-\left(2+\alpha_{k} \tanh \left(\alpha_{k}\right)\right) \cosh \left(\alpha_{y}\right)+\alpha_{y} \sinh \left(\alpha_{y}\right)\right] \sin \left(\alpha_{x}\right) \\
& +\frac{a^{2}}{2 \pi^{2} D} \sum_{k} \frac{(-1)^{\frac{k}{2}}}{k^{2} \cosh \left(\alpha_{k}\right)} B_{k}\left[\alpha_{k} \tanh \left(\alpha_{k}\right) \cosh \left(\alpha_{y}\right)-\alpha_{y} \sinh \left(\alpha_{y}\right)\right] \sin \left(\alpha_{x}\right) \\
& +\frac{a^{2}}{2 \pi^{2} D} \sum_{j} \frac{(-1)^{\frac{j-1}{2}}}{j^{2} \sinh \left(\alpha_{j}\right)} C_{j}\left[\alpha_{j} \frac{\sinh \left(\alpha_{x}\right)}{\tanh \left(\alpha_{j}\right)}-\alpha_{x} \cosh \left(\alpha_{x}\right)\right] \cos \left(\alpha_{y}\right)
\end{aligned}
$$




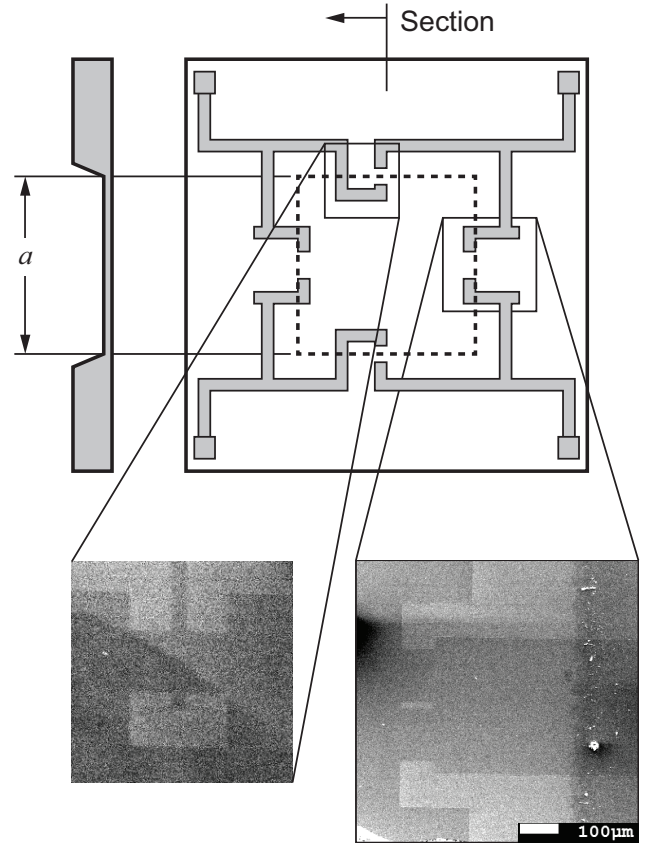

Fig. 1. (Schematic and cross-section of a typical pressure sensing die (20), and micrographs of the piezoresistive elements on the die used.

where $k=2,4,6, \ldots$, and the substitution

$$
\beta=-\frac{(-1)^{\frac{k}{2}}}{16 \alpha_{j}^{5}} \cos \left(\frac{d}{a} \alpha_{k}\right)+\frac{a}{16 \alpha_{k}^{6} d}(-1)^{\frac{k}{2}} \sin \left(\frac{u}{a} \alpha_{k}\right)
$$

has been made for convenience. Again, the coefficients $B_{k}$ and $C_{j}$ may be obtained from the solution of the coupled system

$$
\begin{aligned}
& -\frac{B_{k}}{k}\left(\frac{\alpha_{k}}{\cosh ^{2}\left(\alpha_{k}\right)}+\tanh \left(\alpha_{k}\right)\right)+\frac{8 k}{\pi} \sum_{i} \frac{C_{i}}{i^{3}}\left(1+\frac{j^{2}}{i^{2}}\right)^{-2} \\
= & -12 M \frac{a}{d^{2}} \pi^{2} k \beta_{k}\left(\frac{\alpha_{k}}{\cosh ^{2}\left(\alpha_{k}\right)}-\tanh \left(\alpha_{k}\right)\right)
\end{aligned}
$$

with the index $i=1,3,5, \ldots$ and

$$
\begin{aligned}
& \frac{8}{\pi} j \sum_{i} \frac{B_{i}}{i^{3}}\left(1+\frac{j^{2}}{i^{2}}\right)^{-2}+\frac{C_{j}}{j}\left(\frac{\alpha_{j}}{\sinh ^{2}\left(\alpha_{j}\right)}-\frac{1}{\tanh \left(\alpha_{j}\right)}\right) \\
= & -96 M \frac{a}{d^{2}} \frac{\pi}{j} \sum_{i} i \beta_{i}\left(1+\frac{j^{2}}{i^{2}}\right)^{-2}
\end{aligned}
$$

with the index $i=2,4,6, \ldots$; again, the stresses in the surface of the die are given by substituting the solution to eq. (7) into eq. (5).

This analytical result was validated against a finiteelement numerical solution for a square sensing die having $h / a \sim 7.5 \times 10^{-3}$ (similar to the die tested, and shown in figure 1), under the same assumptions and loading conditions. The differences between the numerical solutions and the analytical one are small (see table I).

Using this solution, the maximum rectified stresses along the edge of the die at $x= \pm a / 2, y=0$ (where

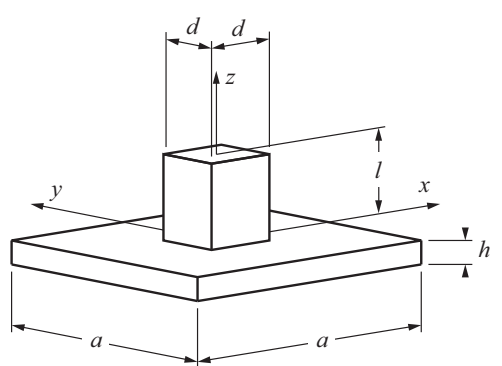

Fig. 2. Schematic of a micropillar attached to a sensing die.

TABLE I

Differences between solution to eq. (7) and discrete numerical results.

\begin{tabular}{|c||c|}
\hline$d / a$ & Error (\%) \\
\hline 0.01 & 2.58 \\
0.025 & 2.05 \\
0.05 & 2.27 \\
0.1 & 2.25 \\
0.2 & 2.50 \\
\hline
\end{tabular}

the sensing element is expected to be located) can be reasonably-well approximated by the expression

$$
\max \left(\sigma_{x}\right) \approx \frac{1.26}{a h^{2}} M
$$

which is notably independent of $d$. If $M$ is selected such that the total rectified force acting on the die is $P a^{2}$, then eq. (11) becomes

$$
\max \left(\sigma_{x}\right) \approx 0.21\left(\frac{d}{h}\right)^{2} P
$$

Comparing eqs. (6) and (12), for equivalent applied loads, the sensitivity of a pressure-sensitive die to direct applied moments by means of a micropillar is expected to vary approximately with $(d / a)^{2}$. On the other hand, if the same pressure $P$ is applied to one side of the pillar, eq. (12) becomes

$$
\max \left(\sigma_{x}\right) \approx 0.63 \frac{d}{a}\left(\frac{l}{h}\right)^{2} P
$$

Since $\sigma \propto(l / h)^{2}, d / a$ was assumed to be of $O(1)$ and $l / h$ is expected to be of $O(10)$ at least, the application of the pillar is expected to enable the sensing die to be sensitive to much smaller applied pressures.

Figures 3 and 4 show the isocontours of normalized deflection

$$
w^{*}(x, y)=\frac{D}{P a^{4}} w(x, y)
$$

and normalized stress along the $x$-axis

$$
\sigma_{x}^{*}=\left(\frac{h}{a}\right)^{2} \frac{1}{P} \sigma_{x}
$$

for a typical die with $h / a=5.6 \times 10^{-3}$ and $d / a=0.22$. Figure 3 shows the case of an applied uniform pressure 

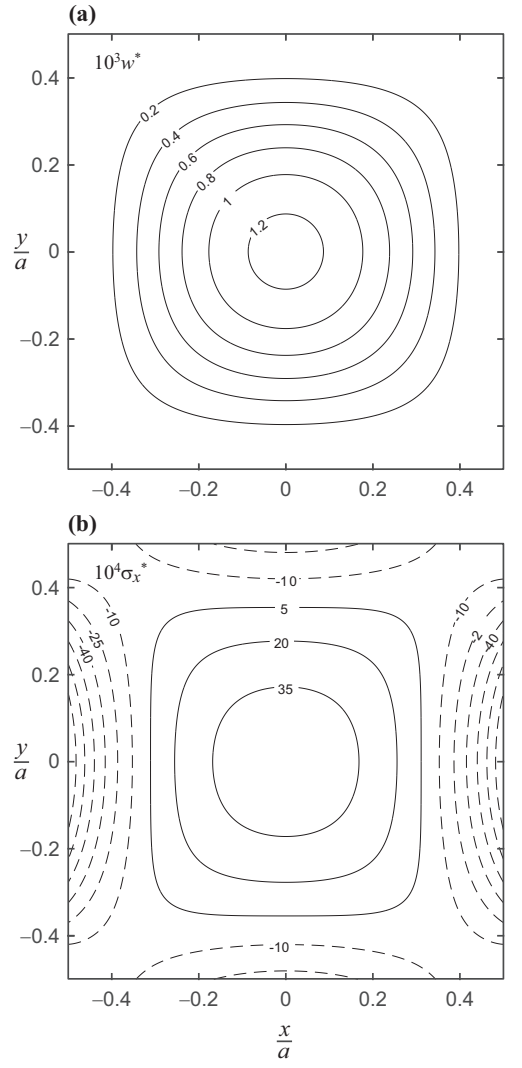

Fig. 3. Isocontours of normalized (a) $w^{*}$ and (b) $\sigma_{x}^{*}$ for the case of applied pressure.

$P$, and figure 4 shows the case of an applied moment. For comparison, equal maximum surface pressures were applied in both cases; this results in significantly lower loads in the case of the pillar, so the the contour levels have been scaled differently. The distribution of horizontal stresses at the boundaries (where the strain sensors are situated) are fairly similar in the two cases, with concentrations at $x= \pm a / 2, y=0$; however, for the case of the applied moment, the sense of $\sigma_{x}$ is reversed for $x<0$.

This solution was developed under two important assumptions: $(a)$ that the moment is acting about the one of the die axes, and $(b)$ that the moment is distributed evenly across that axis. The former is not a strong constraint: because the sensing die is square, the solution cannot be axisymmetric. However, analytical solutions are available along the $x$ and $y$ axes, and approximate solutions at intermediate angles could be obtained by superposition. The second assumption is more constraining, in that the solution approximates a square pillar of side length $d$ with a rectangular one of dimensions $d \times a$ (in order to enable the solution to converge). The error introduced by this assumption becomes small as $d \rightarrow a$. The largest error, at $d \rightarrow 0$, was calculated by numerical simulation and found to be at most a factor of 2.15. This limits the applicability of the analytical solution obtained, but does not affect the order-of-magnitude analysis or the relationships between the parameters.
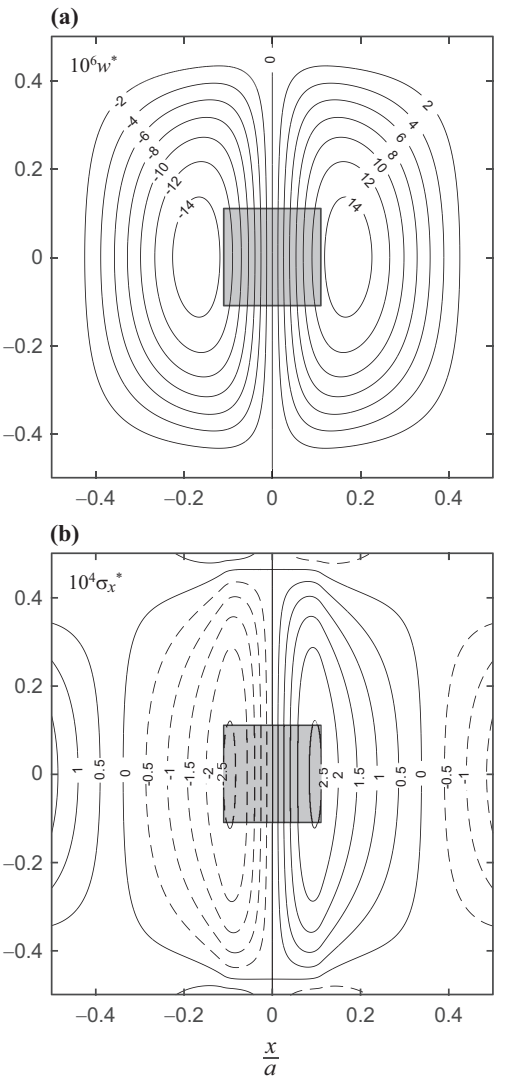

Fig. 4. Isocontours of normalized (a) $w^{*}$ and (b) $\sigma_{x}^{*}$ for the cases of applied moment. Shaded area shows location of pillar.

\section{Measuring moments with a piezoresistive bridge}

With elements positioned at the centres of the four sides of the sensing diaphragm all aligned in the same direction (see figure 1), the normalized resistance change will be proportional to the plane stress, with positive coefficients of proportionality for $R_{1}$ and $R_{3}$, and negative coefficients for $R_{2}$ and $R_{4}$ (as illustrated in figure 5). For maximum sensitivity, pressure sensors are typically designed such that under an applied pressure $\Delta R_{1}=\Delta R_{2}=\Delta R_{3}=\Delta R_{4}$ to within manufacturing tolerance. Then, the voltage measured across the bridge $V_{A B}$ will be given by

$$
V_{A B}=V_{E} \frac{\Delta R}{R}
$$

where $V_{E}$ is the voltage supplied at the top of the bridge. For piezoresistive sensing elements in the configuration shown in figure 1 , the change in resistance is proportional to the strains, as

$$
\frac{\Delta R}{R}=K_{x} \sigma_{x}+K_{y} \sigma_{y}
$$

where $K_{x}$ and $K_{y}$ are the constants of proportionality, and $\sigma_{x}$ and $\sigma_{y}$ can be taken as the maximum stress approximated by eq. (6) for a well-designed sensor. For the case of an applied moment, though, the stress field is not uniform along the edges. If, for example, a moment is applied 


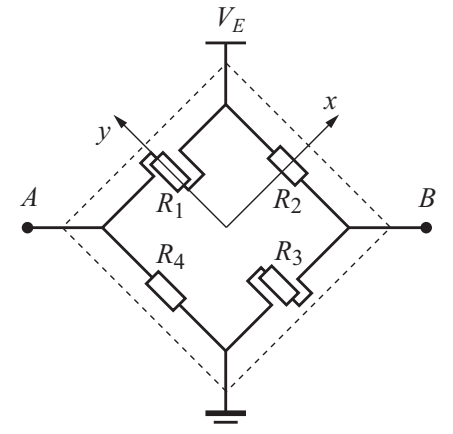

Fig. 5. Schematic of the strain-sensitive resistance bridge on a typical pressure sensing die.

about the $y$-axis in figure 5 , then $\Delta R_{2}=-\Delta R_{4}=\Delta R$, and $\Delta R_{1}=\Delta R_{3}=\Delta R^{\prime}$. Then, assuming $\Delta R \ll R$ and $\Delta R^{\prime} \ll R$,

$$
V_{A B} \sim V_{E}\left(\frac{1}{2} \frac{\Delta R}{R}\right)
$$

Substituting eq. (17) then yields

$$
V_{A B} \sim \frac{1}{2} V_{E} K_{x} \sigma_{x}
$$

When subjected to a moment rather than a pressure, the die will be more sensitive to surface stress. However, with a single output it is impossible to determine the direction; the signal $V_{A B}$ would be the same, for example, whether the moment is applied about the $x$-axis or the $y$-axis.

To resove directions, the voltages at the bridge sides $\left(V_{A}\right.$ and $\left.V_{B}\right)$ may be measured separately relative to ground. Under the same assumptions, then, this would yield two independent measures of surface stress. Then, in the same case of an applied moment about the $y$-axis,

$$
\begin{aligned}
& V_{A}=V_{E} \frac{1-\frac{\Delta R}{R}}{2-\frac{\Delta R}{R}} \\
& V_{B}=V_{E} \frac{1}{2+\frac{\Delta R}{R}}
\end{aligned}
$$

noting again that the stress can be approximated using eq. (12) with eq. (18). Under an applied moment about the $x$-axis, the values for $V_{A}$ and $V_{B}$ will be exchanged, and for other angles, a blending function could be used to interpolate empirically. Although the change in $V_{A}$ and $V_{B}$ under applied moments will be small, they will be sensitive not only to the magnitude of the applied moment, but to both the axis and sense as well.

\section{Experimental demonstration}

To verify the use of off-the-shelf pressure dies to sense micropillar deflection, a small number of demonstration units were constructed and tested.

Cylindrical pillars were fabricated from natural bamboo fibre. Individual fibres were separated and sorted by size, and then selected and rolled to achieve an approximately cylindrical form with diameter $d \sim 0.5 \mathrm{~mm}$. One end face of the fibre was then polished using a fine abrasive. The polished face of the fibre was bonded to the centre of the surface of a pressure sensing die using a bead of epoxy adhesive of approximately the same diameter as the pillar. The adhesive bead was applied, and the pillar positioned and held during the curing process, using a precision threeaxis micro-traversing stage. The fibre was then trimmed to the desired length $l \sim 8 \mathrm{~mm}$. Microphotography images suggested that the surface roughness tolerance on the processed fibres was approximately $\pm 20 \mu \mathrm{m}$.

Honeywell PCE-series silicon membrane-type piezoresistive sensing dies were used, having a reported fullscale pressure sensing range of $3.5 \mathrm{kPa}$, and a nominal bridge resistance $R \sim 5 k \Omega$. The gold contact pads on the sensing dies were reflow-soldered onto an etched FR4 PCB substrate, to secure them in place and provide solder contacts for the signal wires. Vent gaps were deliberately left between the sensing die and the substrate, in order to minimize deflection of the die in response to changes in ambient pressure.

A miniature bridge amplifier circuit featuring two-stage gain and second-order active filtering was developed for this work with particular attention given to attaining the lowest possible noise output. The bridge was powered by a constant voltage supply of $3.0 \mathrm{~V}$ with an output voltage noise of less than $6.5 \mu \mathrm{V}$ RMS. A gain was first applied to the differential voltage across the bridge $\left(V_{B}-V_{A}\right.$ on figure 5) using an INA188 precision instrumentation amplifier, having RFI-filtered inputs and an input noise of only 12 $\mathrm{nV} / \sqrt{\mathrm{Hz}}$ at $1 \mathrm{kHz}$ and $0.25 \mu \mathrm{V}$ peak-to-peak between 0.1 and $10 \mathrm{~Hz}$ (specified at gain factor of 100). The output from the instrumentation amplifier was then sent to an active, buffered second-order Butterworth low-pass filter with gain. The passives required for setting the gain and filter parameters were soldered directly onto the printed circuit board to minimize errors from changes in contact resistance and stray capacitance. The overall gain value, which was typically on the order of several hundred, was selected and fixed to maintain a $-5 \mathrm{~V}$ to $5 \mathrm{~V}$ output without clipping. The analog output signal was then digitized using an 18-bit data acquisition system with a digital resolution of $38 \mu \mathrm{V}$.

When required, Individual bridge-side voltages $V_{A}$ and $V_{B}$ were obtained relative to the bridge ground using a separate, high-precision component analyzer. In all cases, signals were periodically corrected for any drift by rezeroing.

The micropillar sensor assemblies were fixed to a wallmounted turntable in the test section of a $0.6 \mathrm{~m} \times 1 \mathrm{~m}$ wind tunnel facility (see figure 6), such that the pillars were located on the axis of rotation. The pillars were mounted above the tunnel wall boundary layer, and an $\sim 80 \mathrm{~mm} \times 80 \mathrm{~mm}$ end-plate was used to isolate the effects of the supports and cables. The pillars could also be recessed below the surface of the end-plate in order to reduce the exposed height, with the gap between the pillar and the hole through which it protruded minimized. Care was taken during testing to ensure that the ambient 

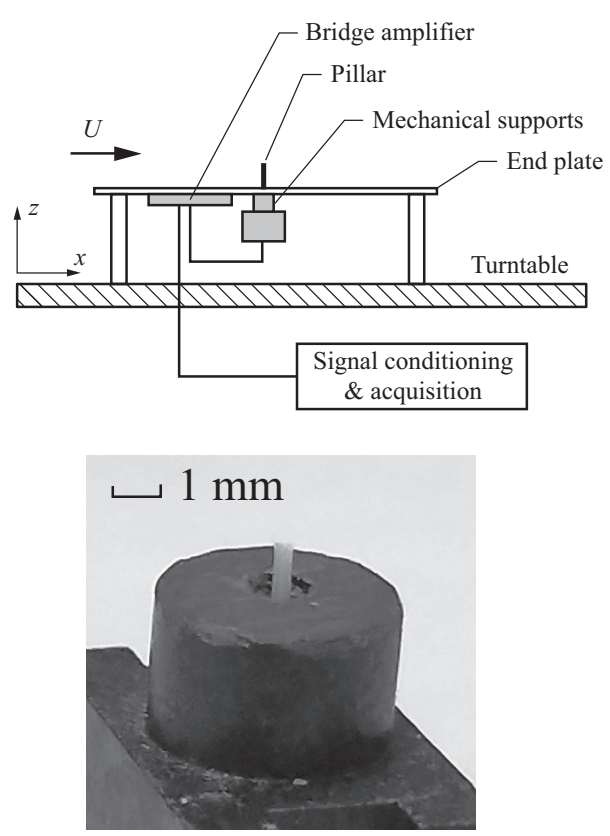

Fig. 6. Schematic of the wind-tunnel test apparatus and image of the pillar sensor.

temperature and lighting remained constant, as both affected the sensor signals. The moment applied to the sensing die by exposing the attached pillar to the flow was estimated by approximating the pillar as a high aspect ratio circular cylinder, and using an accepted empirical relationship between the total aerodynamic drag and Reynolds number $R e=U d / v$, where $v$ is the kinematic viscosity (21).

\section{A. Bridge response to applied moments}

Figure 7 shows the signals obtained over the range $45<\operatorname{Re}<205$, at $\theta=0^{\circ}, 90^{\circ}, 180^{\circ}$ and $270^{\circ}$ (where $\theta$ is the angle subtended between the incident flow velocity vector and the die's arbitrarily-defined $x$-axis) for the case of a pillar having $d=0.5 \mathrm{~mm}$ and $l / d=16$. The results show a clear, linear relationship between $V_{A B}$ and $M$ over this range, as predicted by eqs. (11) and (19). As expected, the sensitivity is unaffected by the sense of the moment. The offsets in the signals are likely the result of a misalignment between the die axes and the tunnel axes.

Figure 8 shows the response of $V_{A B}$ to changes in the flow incidence angle under an applied moment $M=0.69$ $\mu \mathrm{Nm}$ over the range $0^{\circ} \leq \theta \leq 360^{\circ}$. The response is nearly sinusoidal, although with significant distortions around $\theta \sim 0^{\circ}$ and $\theta \sim 180^{\circ}$. These distortions are likely the result of the alignment of the axis of the bending moment with one of the pairs of resistance elements: on the axis, there is likely to be some higher stresses developed at the edge of the membrane as a result of the boundary condition (see figure 4). Some of the irregularity in the behaviour is the result of the poor manufacturing tolerance of the cylinder itself.

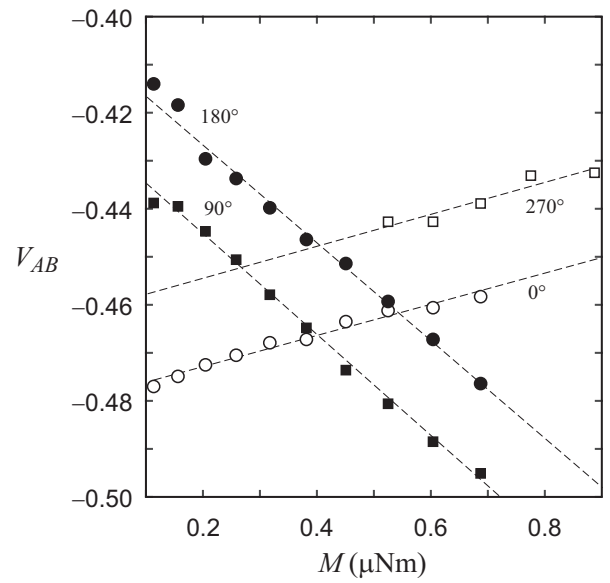

Fig. 7. Response of $V_{A B}$ to applied moments for a pillar with $l / d=16$.

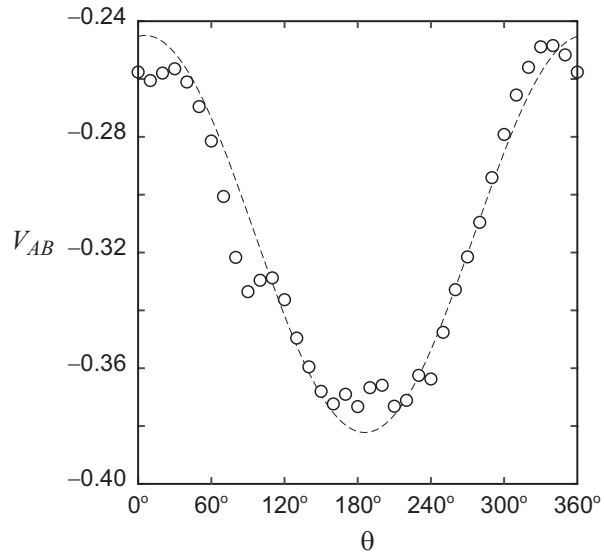

Fig. 8. Response of $V_{A B}$ to changes in flow incidence angle at $M=0.69$ $\mu \mathrm{Nm}$ for a pillar with $l / d=16$. Dashed line shows best-fit sinusoid.

B. Characterization of boundary layers using micropillars

The purpose of the micropillar is to enable the direct measurement of wall shear in turbulent boundary layers, subject to empirical calibration. However, the measurement of wall shear produced by canonical turbulent boundary layers tends to be very uncertain, and no convenient calibration standard was available. Instead, a laminar boundary layer is used here in order to calibrate the pillar system, as it may be taken as an approximation of the viscous sublayer of a turbulent boundary layer. The existence of an analytical solution for laminar boundary layers allows the estimation of $u_{\tau}$ from $U$ with reasonable confidence.

A laminar boundary layer was developed over the endplate (see figure 6) at Reynolds numbers $R e_{x}=U x / v$ (where $x$ is the upstream development length) selected such that $4.7 \times 10^{3}<\operatorname{Re}_{x}<2.4 \times 10^{4}$, well below the critical Reynolds number for transition. For laminar boundary layers, an exact solution for the velocity profile $u(y)$ exists, yielding a boundary layer thickness $\delta$ of

$$
\delta=5 \sqrt{\frac{v x}{U}}
$$


For the range of $U$ and $R e_{x}$ tested, this resulted in boundary layer thicknesses of $1.3<\delta<2 \mathrm{~mm}$ (broadly equivalent to the thickness of sublayer under a turbulent boundary layer having $R e_{x} \sim 10^{6}$ and $U=4 \mathrm{~m} / \mathrm{s}$ ). Exposed pillar heights of $\sim 1 \mathrm{~mm}$ and $\sim 1.3 \mathrm{~mm}$ were therefore used, resulting in $1 \lesssim l / \delta \lesssim 2$. The pillar diameter $d$ was also reduced to $\sim 400 \mu \mathrm{m}$, to reduce three-dimensional effects.

With $u(y)$ and $l$ known, The moment applied on the pillar may be approximated as

$$
M=\int_{0}^{l-l_{0}} \frac{1}{2} \rho u(y)^{2} y d C_{D} d y
$$

where the sensing element is recessed a distance $l_{0}$ below $y=0$, and $C_{D}$ is again empirical. Figure 9(a) shows the signal $V_{A B}$ obtained from the two pillars. The results agree well with those predicted by eqs. (22) and (11), subject to an arbitrary (but identical) gain and offset. For lower $R e$, the pillars were not sensitive to the flow; this is likely the result of residual strain in the pillar assembly incurred when recessed below the surface. The data can equally be rescaled to show the calibration against $\tau$, given that

$$
u_{\tau}=\sqrt{\frac{0.332}{R e_{x}}} U
$$

for the case of a laminar boundary layer. Figure 9(b) shows the same data, and the results appear to collapse well onto eq. (23), again subject to an arbitrary (but consistent) rescaling.

\section{Directionally-resolved measurements}

To demonstrate that the system may be used to resolve the directionality of the applied moment, the signals $V_{A}$ and $V_{B}$ were sampled independently as a function of the orientation of the axis of the applied moment, with $M=0.69 \mu \mathrm{Nm}$. Although small, the signals show a clear, independent behaviour and therefore allow the direction to be inferred (see figure 10; note that the signals have been arbitrarily offset for clarity). The dashed lines on the plot show the arbitrarily-scaled values predicted by eqs. (17) and (11), assuming a sinusoidal variation in $\sigma$ with $\theta$ as an approximation based on the solution of eq. (7). The agreement is reasonable, considering the weakness of the assumptions required (including the shape of the function $\sigma(\theta)$ in particular). Nevertheless, an empirical calibration will be required.

Using the calibration data shown in figure (10), it was possible to return the angle of an unknown applied moment to within $1^{\circ} 50 \%$ of the time, and to within an average of $3^{\circ}$ over all tests.

\section{Discussion}

Commercially available, mass-manufactured pressure sensing dies offer an attractive alternative for the fabrication of sensor-embedded micropillars for use in the characterization of wall-bounded turbulent flows. Although the membrane geometry and the configuration of the strain sensors on the die were driven by the requirement for
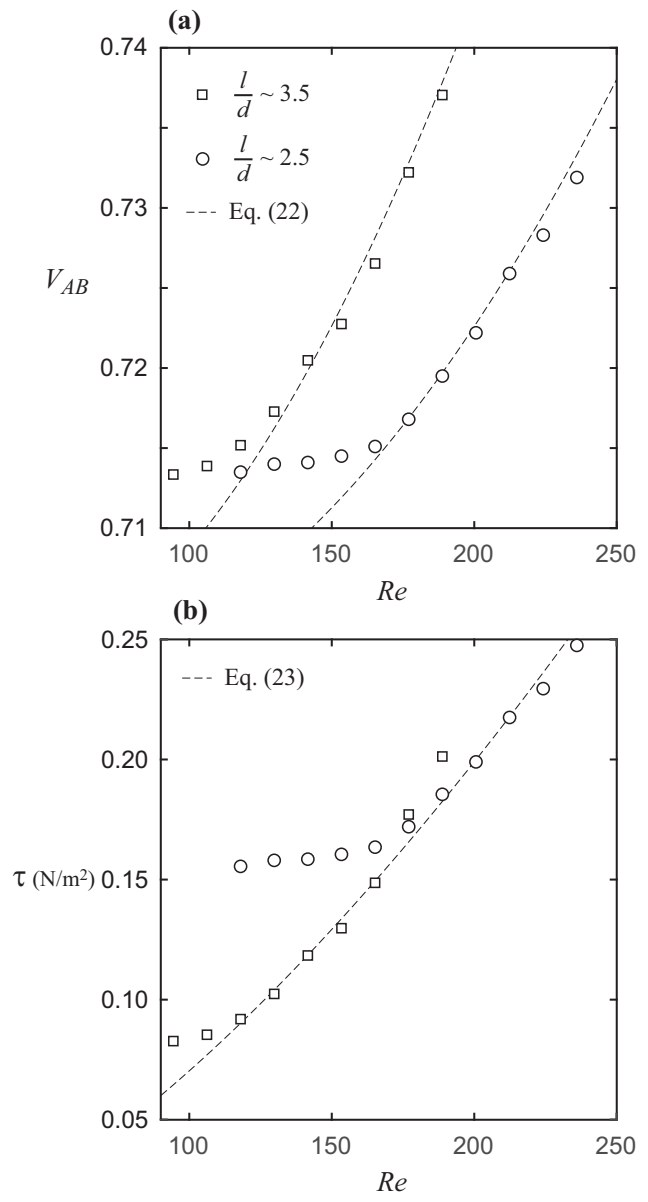

Fig. 9. Signal obtained from pillars having $l / \delta=1$ and 2 in a laminar boundary layer. Dashed line shows eq. (22) or eq. (23).

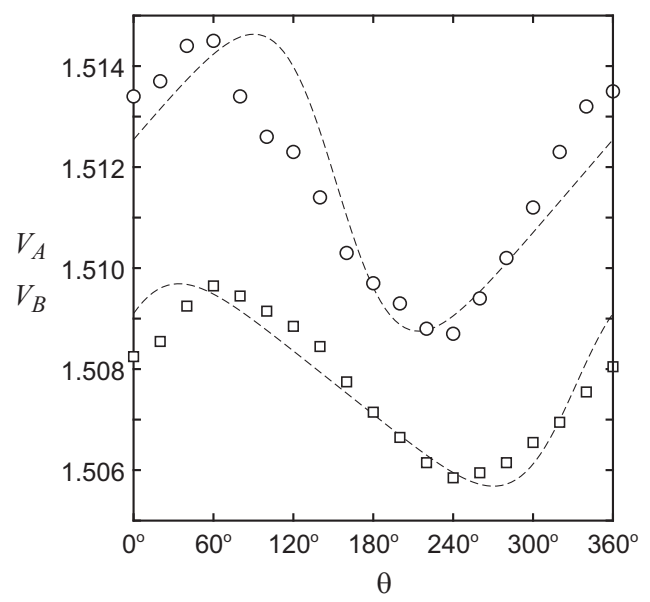

Fig. 10. Response of $V_{A}$ and $V_{B}$ to changes in flow incidence angle at $M=0.69 \mu \mathrm{Nm}$ for a pillar with $l / d=160$. Dashed line shows best-fit to eq. (20) assuming sinusoidal variation in $M$ with $\theta$.

sensitivity to deflection under a uniform applied pressure, the strain fields caused by the deflection of a pillar bonded to the membrane centre can still generate signals sensitive to the applied moment. The strains along the edges of the membrane- where the sensing elements are expected to be 
TABLE II

Comparison of static performance characteristics between the current micropillar system and typical values from a well-developed benchmark optical system (11).

\begin{tabular}{|l|c|c|}
\hline Characteristic & Present system & Ref. $(11)$ \\
\hline Reported $\tau$ range $(\mathrm{Pa})$ & 0.25 & 1.25 \\
Est. $\tau$ repeatability $(\% \mathrm{FS})$ & 2.2 & 2.9 \\
$d(\mu \mathrm{m})$ & $400 \sim 500$ & $20 \sim 45$ \\
$l(\mu \mathrm{m})$ & $1000 \sim 1300$ & $100 \sim 400$ \\
\hline
\end{tabular}

positioned- will also be nearly linearly proportional to the applied moments.

The concept was first validated by means of testing a pressure sensing die fitted with a pillar by applying loads to the pillar using aerodynamic drag (as the ability to repeatably apply moments with a resolution as low as $10^{-8} \mathrm{Nm}$ was required). The sensor responded linearly to the applied moments, with sensitivities of $\sim 0.07 \mathrm{~V} / \mu \mathrm{Nm}$ in bridge configuration, using an amplifier with a gain of 40. The system also responded to the sense of the applied moment. The ability of the pillars to characterize a boundary layer was also confirmed using a laminar boundary layer to approximate the viscous sublayer of a turbulent boundary layer. Although it is acknowledged that this is a very weak approximation, for the purposes of validating the calibration of the system in near-wall flows, it is sufficient. The sensor exhibited a minimum sensitivity, attributed to residual strains incurred in the process of installation. Once calibrated, the pillars were successfully able to return the dimensional wall shear.

When used in bridge configuration for maximum sensitivity, however, the sensor returned only a single measurement. Consequently, it was impossible to resolve directionality of the applied moment. Sensitivity to directionality was achieved, however, by measuring the signal at the two sides of the bridge independently.

This embedded-sensor micropillar system offers sufficient sensitivity for use in wall shear measurement in the laboratory environment, for either boundary layer characterization or control. The sensitivity and range of the system compares well against well-validated, mature optical micropillar technology (see Table II). By repurposing a mass-market pressure sensing die, the unitto-unit variability in the sensor characteristics and the unit cost are sufficiently low to enable the fabrication of very-large arrays.

\section{Conclusion}

A novel technique for producing strain-sensitive micropillars has been proposed. It has been shown that if a micropillar is bonded to a membrane, the strain field in the membrane resulting from forces applied to the pillar will be detectable by the strain sensors etched onto typical, commercially-available pressure sensing dies. Pillars with lengths ranging between $1 \mathrm{~mm}$ and $8 \mathrm{~mm}$ were produced. The pillars were shown to be sensitive to both the magnitude and direction of the applied loads depending on the signals sampled, and their use in the measurement of wall shear has been demonstrated.

\section{Acknowledgments}

This work was supported in part by a grant from the China Scholarship Council and by the European Commission Erasmus+ programme. Data are available online using DoI 10.15126/surreydata.7330955.

\section{References}

[1] K. Winter, "An outline of techniques available for the mesaurement of skin friction in turbulent boundary layers," Prog. Aerosp. Sci., vol. 18, pp. 1-57, 1977.

[2] H. H. Fernholz, G. Janke, M. Schober, P. Wagner, and D. Warnack, "New developments and applications of skin-friction measuring techniques," Meas. Sci. Technol., vol. 7, pp. 1396-1409, 1996.

[3] C. Brücker, "Evidence of rare backflow and skinfriction critical points in near-wall turbulence using micropillar imaging," Phys. Fluids, vol. 27, no. 3, p. 031705, 2015.

[4] J. H. Preston, "The determination of turbulent skin friction by pitot tubes," J. R. Aeronaut. Soc., vol. 58, pp. 109-121, 1954.

[5] I. P. Castro and M. Dianat, "Pulsed wire anemometry near walls," Exp. Fluids, vol. 8, pp. 343-352, 1990.

[6] C. F. Colebrook, "Turbulent flow in pipes with particular reference to the transition region between region between the smooth- and rough-wall laws." J. Inst. Civil Eng., vol. 11, pp. 133-156, 1939.

[7] F. H. Clauser, "The turbulent boundary layer." Adv. Appl. Mech., vol. 4, pp. 1-51, 1956.

[8] I. P. Castro, "Rough-wall boundary layers: mean flow universality," J. Fluid Mech, vol. 585, pp. 469-485, 2007.

[9] D. M. Birch and J. F. Morrison, "Similarity of streamwise velocity component in very-rough-wall channel flow," J. Fluid Mech., vol. 668, pp. 174-201, 2011.

[10] S. Große and W. Schröder, "Mean wall-shear stress measurements using the micro-pillar shear-stress sensor mps ${ }^{3}$," Meas. Sci. Technol., vol. 19, p. 015403, 2008.

[11] E. P. Gnanamanickam, B. Nottebrock, S. Große, J. P. Sullivan, and W. Schröder, "Measurement of turbulent wall shear-stress using micro-pillars," Meas. Sci. Technol., vol. 24, p. 124002, 2013.

[12] J. Paek and J. Kim, "Microsphere-assisted fabrication of high aspect-ratio elastomeric micropillars and waveguides," Nature communications, vol. 5, no. 3324, pp. 1-8, 2014.

[13] J. M. Engel, J. Chen, C. Liu, and D. Bullen, "Polyurethane rubber all-polymer artificial hair cell sensor," J. MEMS, vol. 15, no. 4, pp. 729-735, 2006.

[14] H. Devaraj, J. Travas-Sejdic, R. Sharma, N. Aydemir, D. Williams, E. Haemmerle, and K. Aw, "Bio-inspired flow sensor from printed PEDOT:PSS micro-hairs," 
Bioinspiration and Biomimetics, vol. 10, no. 1, p. 016017, 2015.

[15] S. Wang, D. Feng, C. Hu, and P. Rezai, "The simple two-step polydimethylsiloxane transferring process for high aspect ratio microstructures," J. Semiconductors, vol. 39, no. 8, p. 086001, 2018.

[16] Q. Li, R. Dhakal, and J. Kim, "Microdroplet-based on-demand drawing of high aspect-ratio elastomeric micropillar and its contact sensing application," Sci. reports, vol. 7, p. 17009, 2017.

[17] M. Sheplak, L. Cattafesta, and T. Nishida, "MEMS shear stress sensors: promise and progress," in Proceedings of the 24th AIAA Aerodynamic Measurement Technology and Ground Testing Conference, 2004, pp. 2004-2606.

[18] I. Marusic, "On the role of large-scale structures in wall turbulence," Phys. Fluids, vol. 13, no. 3, pp. 735-743, 2001.

[19] S. Timoshenko, Theory of plates and shells. McGraw-Hill, 1959.

[20] D. J. Maurer, "Piezoresistive pressure transducer with a conductive elastomeric seal," U.S. Patent 5,184,107, 1993.

[21] H. Schlichting, Boundary-Layer Theory, 4th Ed. McGraw Hill, 1960. 